\title{
Vimentin expression status is a potential biomarker for brain metastasis development in EGFR-mutant NSCLC patients
}

\author{
Rattanawadee Teocharoen ${ }^{1}$, Komkrit Ruangritchankul ${ }^{2}$, Chanida Vinayanuwattikun ${ }^{1}$, \\ Virote Sriuranpong ${ }^{1}$, Piyada Sitthideatphaiboon ${ }^{1}$ \\ ${ }^{1}$ Division of Medical Oncology, Department of Medicine, Faculty of Medicine, Chulalongkorn University and The King Chulalongkorn Memorial \\ Hospital, Bangkok, Thailand; ${ }^{2}$ Department of Pathology, Faculty of Medicine, Chulalongkorn University and The King Chulalongkorn Memorial \\ Hospital, Bangkok, Thailand \\ Contributions: (I) Conception and design: R Teocharoen, P Sitthideatphaiboon, V Sriuranpong; (II) Administrative support: C Vinayanuwattikun; \\ (III) Provision of study materials or patients: K Ruangritchankul, C Vinayanuwattikun; (IV) Collection and assembly of data: $\mathrm{R}$ Teocharoen, K \\ Ruangritchankul, P Sitthideatphaiboon; (V) Data analysis and interpretation: R Teocharoen, K Ruangritchankul, P Sitthideatphaiboon; (VI) \\ Manuscript writing: All authors; (VII) Final approval of manuscript: All authors. \\ Correspondence to: Piyada Sitthideatphaiboon, MD. Division of Medical Oncology, Department of Medicine, Faculty of Medicine, Chulalongkorn \\ University and The King Chulalongkorn Memorial Hospital, 1873 Henry Do-Nant Rd, Pathumwan, Bangkok 10330, Thailand. \\ Email: Piyada.Si@chula.ac.th.
}

Background: Despite advances in systemic therapy and improvements in survival for advanced epidermal growth factor receptor $(E G F R)$ mutant non-small cell lung cancer (NSCLC), brain metastasis (BM) remains a poor outcome. Previous studies on risk factors for BM occurrence included unselected patients and biomarker prediction of BM in these populations were not well studied. We aimed to identify the role of epithelial mesenchymal transition (EMT) marker and clinical factors predicting BM in EGFR-mutant NSCLC patients.

Methods: Advanced EGFR-mutant NSCLC patients in the King Chulalongkorn Memorial Hospital from January 2013 to December 2017 were included. Vimentin expression was assessed by immunohistochemistry. The correlation between vimentin expression and factors associated with BM occurrence was analyzed by univariate and multivariate analyses.

Results: 304 patients were enrolled. Of these, 149 patients (49\%) developed BM. In multivariate analysis, the occurrence of BM was associated with age $<60$ years, metastatic disease at diagnosis, and 3 or more metastatic sites. Moreover, positive vimentin expression was also found more common in patients with BM than those without BM (52.4\% vs. 27.6\%, respectively) and predicted overall BM development in EGFRmutant patients (OR 2.53, 95\% CI, 1.11-5.77; $\mathrm{P}=0.027$ ). Overall survival (OS) was shorter in vimentin ${ }^{\text {positive }}$ group than in vimentin ${ }^{\text {negative }}$ group. Median OS was 20.0 months (95\% CI, 14.51-25.51) and 30.9 months (95\% CI, 20.99-40.84), respectively (HR, 1.57; P=0.04).

Conclusions: Younger patients with EGFR-mutant NSCLC who had high disease burden were more likely to develop BM. Vimentin served as a biomarker for predicting BM and poor prognostic factor in EGFRmutant patients. EMT pathway may be considered as a therapeutic target in these high-risk populations.

Keywords: Non-small cell lung cancer (NSCLC); epidermal growth factor receptor (EGFR) mutation; epithelial mesenchymal transition (EMT); vimentin, brain metastasis (BM)

Submitted Sep 05, 2020. Accepted for publication Dec 23, 2020.

doi: $10.21037 /$ tlcr-20-1020

View this article at: http://dx.doi.org/10.21037/tlcr-20-1020 


\section{Introduction}

Non-small cell lung cancer (NSCLC) poses a major health problem throughout the world and also in Thailand $(1,2)$. Only $19 \%$ of all patients with lung cancers are alive 5 years or more after diagnosis (3). Despite newer targeted agents improving the systemic control of malignancy and hence survival, the incidence of brain metastasis (BM) has still increased. Approximately 10\% of NSCLC patients develop $\mathrm{BM}$ at presentation, and approximately $40 \%$ of all patients develop BM subsequently (4). Incidence is higher in patients, especially whose cancers harbor epidermal growth factor receptor $(E G F R)$ mutation, in whom up to $50-60 \%$ will develop BM over the course of their disease (5-8). The association between the EGFR mutation status and BM in patients with NSCLC has been reported $(5,8)$. Patients with $E G F R$ mutations were more likely to develop BM than those with $E G F R$ wild type, especially during the course of the disease (9). It remains unclear whether this is because these patients have longer survival times and thus, more time to develop BM, whether there are selective pressure and poor central nervous system (CNS) penetration of systemic therapies, or whether these mutation-driven cancers have biologic features that predispose towards progression and growth within the CNS. Previous studies on risk factors for the development of BM in NSCLC including younger age (8,10-13), female gender (12), non-squamous cell carcinoma (11-14), and more advanced in tumor and nodal stage $(8,10,11,14)$ have been reported, however, most of these studies included unselected patients with NSCLC. Biomarker prediction for BM in these populations is not well studied.

Several studies suggested that molecular factors play an important role in contributing to $\mathrm{BM}$, such as genes involved in cell adhesion, extravasation, metabolism, and cellular signaling (15). Epithelial mesenchymal transition (EMT), a process by which epithelial cells lose their cell polarity and cell-cell adhesion and gain migratory and invasive properties to become mesenchymal stem cells, play a role in the initiation of metastasis. Accumulating evidence has indicated that vimentin is critical for the progression and prognosis of lung cancer (16). Furthermore, activation of EGFR expression promoted EMT phenotype in various cancer cell lines, including lung cancer $(17,18)$. Although the correlation between EGFR mutation and BM has been widely studied $(9,13,19,20)$, data concerning the association of EMT status and BM development are scare and underlying mechanisms of BM progression in these patients remain poorly understood.
Therefore, we aimed to identify the factors associated with BM in EGFR-mutant NSCLC and identify the patients at higher risk for BM development for earlier detection and treatment as well as characterizing the role of EMT marker as a biomarker that can predict the occurrence of BM in EGFR-mutant NSCLC. We present the following article in accordance with the REMARK reporting checklist (available at http://dx.doi.org/10.21037/tlcr-20-1020).

\section{Methods}

\section{Study population}

This retrospective study enrolled patients who were diagnosed with recurrent/metastatic NSCLC at King Chulalongkorn Memorial Hospital (KCMH) over a period of 5 years (January 1, 2013, to December 31, 2017) and had complete patient medical records on key exposure and outcome variables. The main inclusion criteria were adults aged 18 or older with cytology or histologically confirmed NSCLC who had EGFR testing results. The patients were excluded if they had more than one primary cancer, unknown EGFR mutation status, had anaplastic lymphoma kinase (ALK) rearrangement or other mutations, and incomplete follow up data. The presence of BM was confirmed by brain radiography, either by computed tomography (CT) or magnetic resonance imaging (MRI). Patients were categorized into initial BM (inBM) if $\mathrm{BM}$ was identified at presentation and subsequent BM (subBM) if $\mathrm{BM}$ was identified in patients who had negative brain imaging at baseline and were imaged to identify BM when $\mathrm{BM}$ associated neurologic symptoms/signs occurred or BM found during or after treatment. The variables include age, gender, Eastern Cooperative Oncology Group (ECOG) performance status (PS), smoking status, histology, initial stage at diagnosis, number of metastatic sites, EGFR mutation subtypes, and treatment history were collected. EGFR mutations (including G719X in exon 18, exon 19 deletion, T790M in exon 20, and L858R and L861Q in exon 21) were performed by cobas ${ }^{\circledR}$ EGFR Mutation Test v2 kit according to the manufacture's protocol. This study was approved by the Institutional Review Board of the Faculty of Medicine at Chulalongkorn University. (No. 267/62). For this retrospective study, the written informed consent from patients was waived per the IRB, and the study was performed following the Health Insurance Portability and Accountability Act and the Declaration of Helsinki (as revised in 2013). 


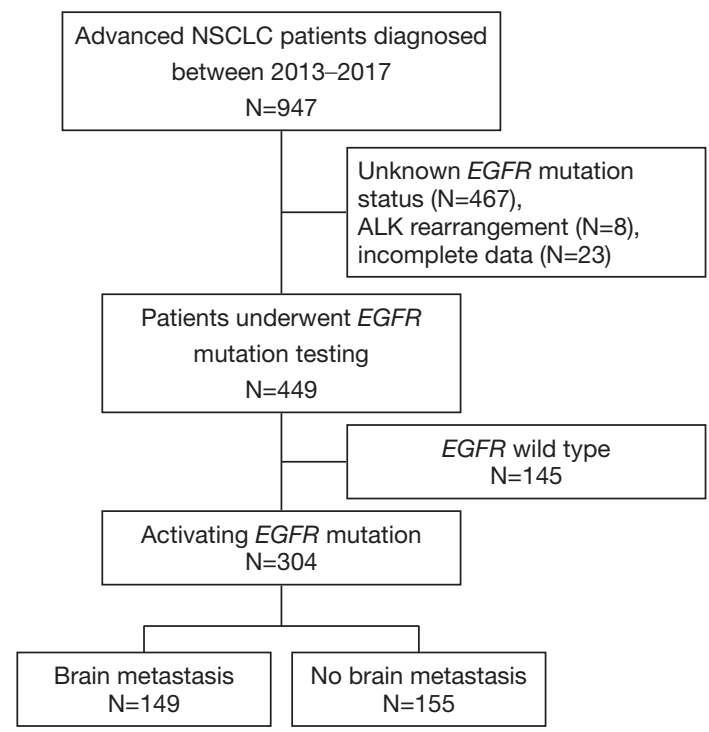

Figure 1 Flow chart of study. Of the 449 non-small cell lung cancer (NSCLC) patients, 304 patients harbored activating epidermal growth factor receptor $(E G F R)$ mutation were included in the final analysis.

\section{Immunobistochemistry}

Formalin-fixed and paraffin-embedded (FFPE) tumor samples from the histopathological files of the Department of Pathology, KCMH were retrospectively analyzed. Twomicron thick FFPE tissue sections on charged glass slides were prepared per standard protocol for IHC. Epitope retrieval was performed on the Dako PT link (Dako, Denmark) and immunostaining was performed using automated staining systems, DakoAutostainer Link48 (Dako, Denmark) with antibodies against vimentin antibody (Monoclonal Mouse anti-Human Vimentin, clone V9, RTU, Cat no; IR630, Dako Denmark). In accordance with similar thresholds used in previous studies (21), a value of $\geq 10 \%$ positive tumor cells independent of intensity was chosen to define positive expression of vimentin . Briefly, the staining intensity was determined by cytosolic staining for vimentin. Vimentin expression that was equal to or more than $10 \%$ of tumor cells with cytoplasmic staining intensity of $+1,+2$ or +3 was categorized as positive. All slides were evaluated by a pathologist (K.R) who was blinded from patient outcomes.

\section{Statistical analysis}

Categorical variables were summarized by frequencies and percentages while continuous variables were reported by the median and interquartile range (IQR). Clinicopathologic factors and treatment outcomes were analyzed in correlation with BM status using Chi-square or Fisher exact test as appropriate. The univariate and multivariate analysis assessed factors associated with the development of BM and analyzed by odds ratio (OR). Overall survival (OS) was defined from the date of recurrent or metastatic NSCLC diagnosis to the date of death or the last contact. Patients who were not deceased were censored on December 31, 2019. Time-to-event was analyzed using the Kaplan-Meier method and was compared between groups by the log-rank test. Hazard ratios (HR) and corresponding 95\% confidence intervals $(95 \% \mathrm{CI})$ were calculated. The $\mathrm{P}$ value of less than 0.05 was considered statistically significant. All statistical analyses were conducted using GraphPad Prism version 8.00 for Windows (GraphPad Software, La Jolla, California, USA) and SPSS 23.0 (SPSS Inc, Chicago, Illinois, USA).

\section{Results}

\section{Patient characteristics of the study population}

449 patients were identified. Of these, 304 patients (67.7\%) who had EGFR mutations were analyzed (Figure 1). Baseline characteristics are summarized in Table 1. The median age of the patients was 63 years (IQR 55-70.8), mostly female (65.8\%), good ECOG PS 0 to $1(86.8 \%)$ and never smokers $(76.6 \%)$. The majority of the patients were diagnosed as adenocarcinoma (93.8\%), metastatic disease at 
Table 1 Baseline characteristics in EGFR-mutant patients ( $N=304)$

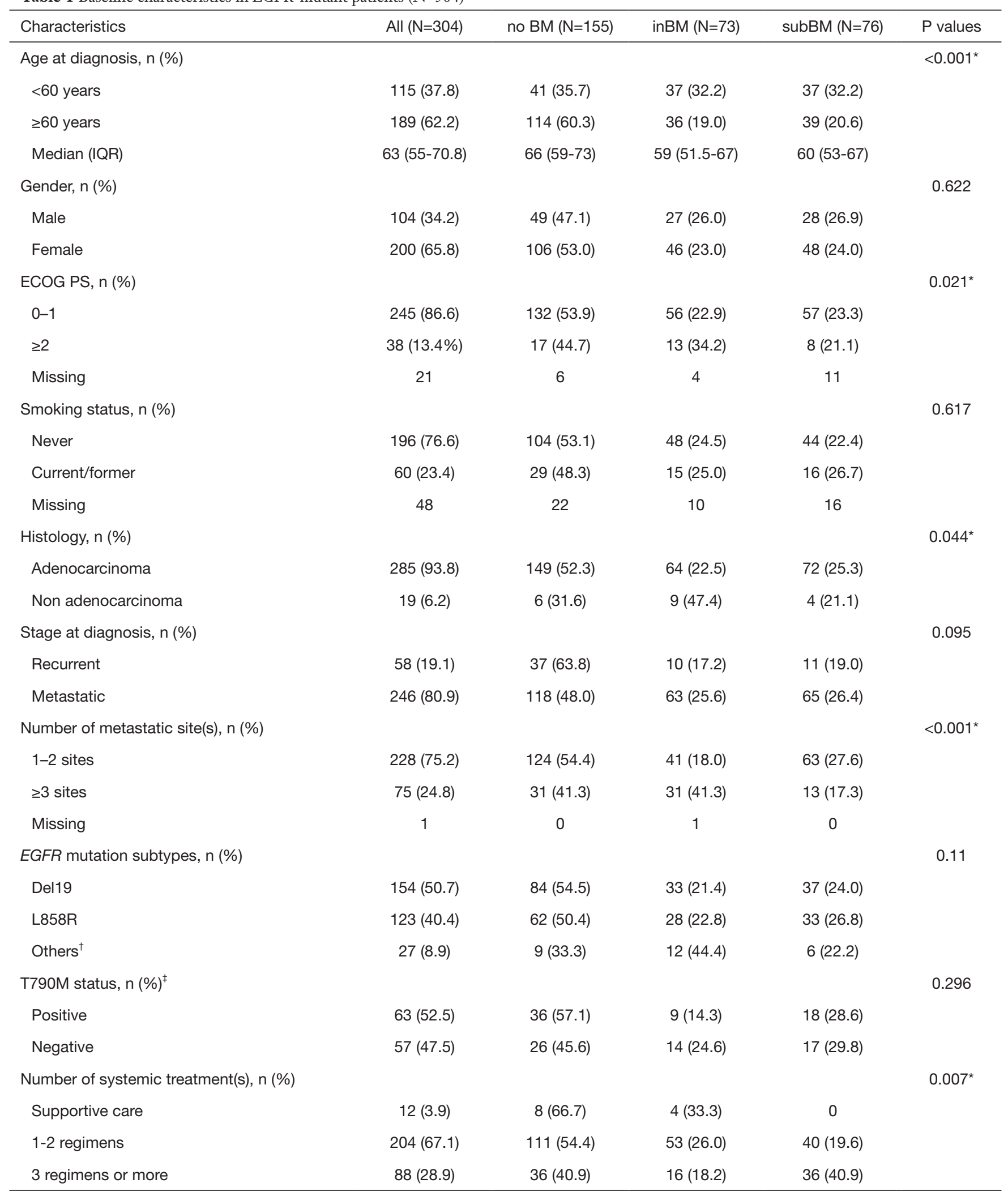

Table 1 (continued) 
Table 1 (continued)

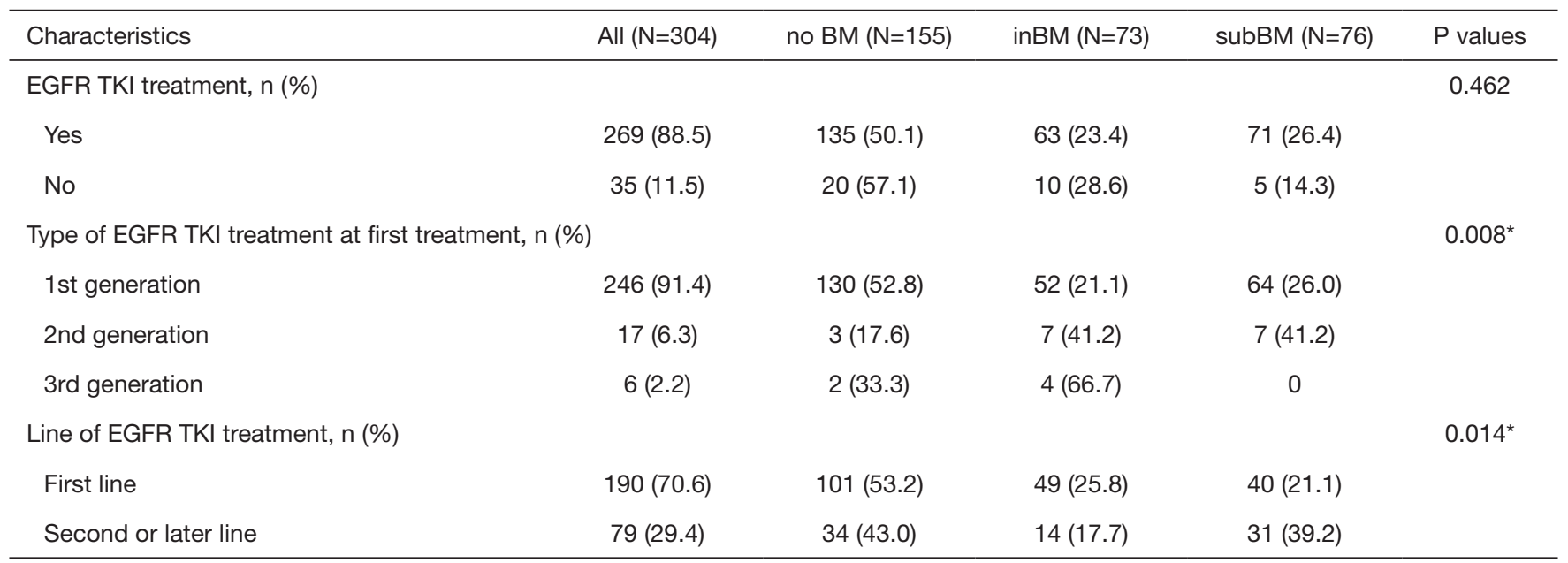

${ }^{\dagger}$, Other EGFR mutation subtypes included exon 21 insertion $(n=8)$, L861G $(n=6), G 719 X(n=5), S 768 I(n=1)$ and any EGFR mutations $(n=7)$, respectively. ${ }^{\ddagger}$, Only 120 patients who progressed after EGFR TKIs treatment were further tested for secondary T790M mutation; ${ }^{*}, \mathrm{P}<0.05$. EGFR, epidermal growth factor receptor; BM, brain metastasis; inBM, initial brain metastasis; subBM, subsequent brain metastasis; IQR, interquartile range; ECOG PS, Eastern Cooperative Oncology Group Performance Status; TKI, tyrosine kinase inhibitor.

diagnosis (80.9\%), and 1 or 2 metastatic sites (75.2\%). Exon 19 deletion $(\mathrm{n}=154)$ and L858R mutation $(\mathrm{n}=123)$ were the most common EGFR mutation subtypes, accounting for $91.1 \%$ of the patients. Other EGFR mutation subtypes included exon 21 insertion ( $\mathrm{n}=8)$, L861G $(\mathrm{n}=6), \mathrm{G} 719 \mathrm{X}$ $(\mathrm{n}=5)$, S768I $(\mathrm{n}=1)$ and any EGFR mutations $(\mathrm{n}=7)$, respectively. Sixty-seven percent of patients received 1 or 2 lines of systemic treatment for the advanced stage of the disease. Of these, 269 patients (88.5\%) received EGFR-TKIs during the course of the disease and 190 patients $(70.6 \%)$ were treated with EGFR-TKIs as first-line treatment. 91.4\% of patients received $1^{\text {st }}$ generation EGFR TKI as the first EGFR TKI treatment. A total of 120 patients (45.6\%) who progressed after $E G F R$-TKIs treatment were further tested for secondary T790M mutation and 63 patients (52.5\%) were found to have the T790M mutation. Of these, 58 patients received osimertinib, $3^{\text {rd }}$ generation of EGFR TKIs, as subsequent treatment.

\section{Factors associated with the development of BM in patients with EGFR-mutant NSCLC}

The median follow-up was 46.42 months (95\% CI, 41.34-51.51), 73 patients (24\%) experienced BM at diagnosis and 76 patients (25\%) developed subBM. Baseline characteristics are reported in Table 1.

The clinicopathological factors that were significantly associated with the overall occurrence of $\mathrm{BM}$ included age $<60$ years (OR 2.74, 95\% CI, 1.69-4.43, $\mathrm{P}<0.001$ ), metastatic disease at diagnosis (OR 1.91, 95\% CI, 1.05-3.45, $\mathrm{P}=0.032$ ), and 3 or more metastatic sites (OR 1.69 , 95\% CI, 0.99-2.87, $\mathrm{P}=0.05)$. Multivariate analyses showed that only age $<60$ years was statistically significantly associated with $\mathrm{BM}$ occurrence. However, there was no difference in BM occurrence between the exon 19 deletion and L858R mutation (Table 2).

Interestingly, factors associated with the development of BM in patients with EGFR-mutant NSCLC differed between those who experienced $\mathrm{BM}$ at diagnosis and those who developed BM subsequently. EGFR-mutant patients who had inBM were more likely to be younger $(<60$ years) (OR 2.85, 95\% CI, 1.59-5.11, $\mathrm{P}<0.001)$, with nonadenocarcinoma histology (OR 3.49, 95\% CI, 1.19-10.21, $\mathrm{P}=0.022$ ), 3 or more metastatic sites (OR $3.02,95 \% \mathrm{CI}$, 1.64-5.56, $\mathrm{P}<0.001)$ and had uncommon $E G F R$ mutation subtype (OR 3.19, 95\% CI, 1.27-7.96, $\mathrm{P}=0.013$ ) compared to patients without $\mathrm{BM}$. Multivariate analyses revealed that younger patients ( $<60$ years) (OR 2.81, 95\% CI, 1.51-5.24, $\mathrm{P}=0.001)$ and higher disease burden $(\geq 3$ metastatic sites) (OR 3.00, 95\% CI, 1.57-5.74, $\mathrm{P}=0.001$ ) were statistically significantly associated with inBM development. While only age $<60$ years (OR 2.63, 95\% CI, 1.47-4.69, $\mathrm{P}=0.001$ ) was associated with subBM compared to those without BM (Table 3). 
Table 2 Univariate and multivariate analysis for clinicopathological factors associated with brain metastasis in EGFR-mutant patients

\begin{tabular}{|c|c|c|c|c|}
\hline Covariate $^{\dagger}$ & OR $(95 \% \mathrm{Cl})$ & $P$ value & OR $(95 \% \mathrm{Cl})$ & $P$ value \\
\hline Gender (Male/female) & $1.26(0.78-2.03)$ & 0.331 & & \\
\hline ECOG PS ( $\geq 2 / 0-1)$ & $1.44(0.72-2.86)$ & 0.295 & & \\
\hline Histology (Non-ADC/ADC) & $2.37(0.87-6.42)$ & 0.089 & $1.99(0.69-5.70)$ & 0.199 \\
\hline Stage at diagnosis (M1/M0) & $1.91(1.05-3.45)$ & $0.032^{\star}$ & $1.83(0.98-3.41)$ & 0.058 \\
\hline No. metastatic site $(\geq 3 /<3)$ & $1.69(0.99-2.87)$ & $0.050^{\star}$ & $1.65(0.95-2.87)$ & 0.075 \\
\hline EGFR subtype (Del19/L858R) & $1.18(0.73-1.90)$ & 0.493 & & \\
\hline
\end{tabular}

${ }^{\dagger}$, Category after the slash (/) was set as reference category. ${ }^{*}, \mathrm{P}<0.05$. EGFR, epidermal growth factor receptor; ADC, adenocarcinoma; ECOG PS, Eastern Cooperative Oncology Group Performance Status; M1, metastatic disease; M0, recurrent disease; OR, odds ratio.

\section{Systemic treatment may contribute to the occurrence of subBM in patients with EGFR-mutant NSCLC}

We analyzed whether systemic treatment is associated with subBM occurrence. Among 231 patients with EGFRmutated NSCLC without BM at diagnosis, 223 patients $(96.5 \%)$ received systemic treatment for the advanced stage of the disease. Of these, 206 patients $(89.2 \%)$ received EGFR-TKIs during the course of the disease and 141 patients (68.4\%) were treated with EGFR-TKIs as first-line treatment. 54 of 101 patients who progressed after EGFRTKIs treatment were found to have secondary T790M mutation and 57 patients received $3^{\text {rd }}$ generation of EGFR TKIs as subsequent treatment.

Patients who received 3 lines or more of systemic treatment (OR 2.84, 95\% CI, 1.58-5.12, $\mathrm{P}<0.001)$ and did not receive $E G F R$-TKI as first-line treatment (OR 2.30, 95\% CI, 1.25-4.23, $\mathrm{P}=0.007$ ) were associated with subBM. However, these treatment factors were not statistically significantly associated with subBM occurrence after adjusting for other clinicopathological factors (Table S1). Outcomes of EGFR-TKI were also analyzed by the time to subBM (TTSBM). Treatment of EGFR-TKIs had longer time to subBM than those patients who did not receive EGFR-TKIs (median TTSBM was 51.78 months vs. 26.61 months, $\mathrm{P}=0.002$; Figure $\mathrm{S} 1$ ). Cox regression analysis was performed on the factors that would correlate with TTSBM. Multivariate analyses revealed that the treatment of EGFR TKIs could delay the occurrence of subBM more than those who did not receive EGFR-TKIs (HR 2.18, 95\%
CI, 1.18-4.02, $\mathrm{P}=0.013$; Table S2).

\section{Outcome of BM in patients with EGFR-mutant NSCLC}

At data cut-off on December 31, 2019, the median followup time was 46.42 months (95\% CI, 41.34-51.51) and 80 patients $(26.3 \%)$ survived to the last contact. The median OS of the overall study cohort was 22.97 months (95\% CI, 20.98-24.95). Patients with BM had a significantly shorter OS than patients without BM (median OS was 22.44 months (95\% CI, 19.76-25.12) vs. 24.18 months (95\% CI, 20.41-27.95), respectively; HR 1.48; 95\% CI, $1.14-1.93, \mathrm{P}=0.004$; Figure $2 A$ ).

Of the 304 patients with EGFR-mutant NSCLC, 268 patients (88.2\%) received EGFR TKIs whereas 36 patients (11.8\%) did not. Patients without BM who received EGFR TKIs had longer survival than those with BM who received EGFR TKIs, and those with/without BM but not received EGFR TKIs (median OS were 25.13, 22.96, 12.22 and 14.39 months, respectively; $\mathrm{P}<0.001$; Figure $2 B$ ).

\section{Vimentin expression status as one of EMT marker predicts the development of BM in patients with EGFR-mutant NSCLC}

To validate whether vimentin expression status as a predictive marker for $\mathrm{BM}$ occurrence, we next analyzed vimentin expression by IHC on 190 available tumor specimens according to EGFR mutation status. Baseline characteristics of these 190 patients are listed in Table S3. 
Table 3 Univariate and multivariate analysis for the occurrence of BM at diagnosis and subsequent BM in EGFR mutant patients

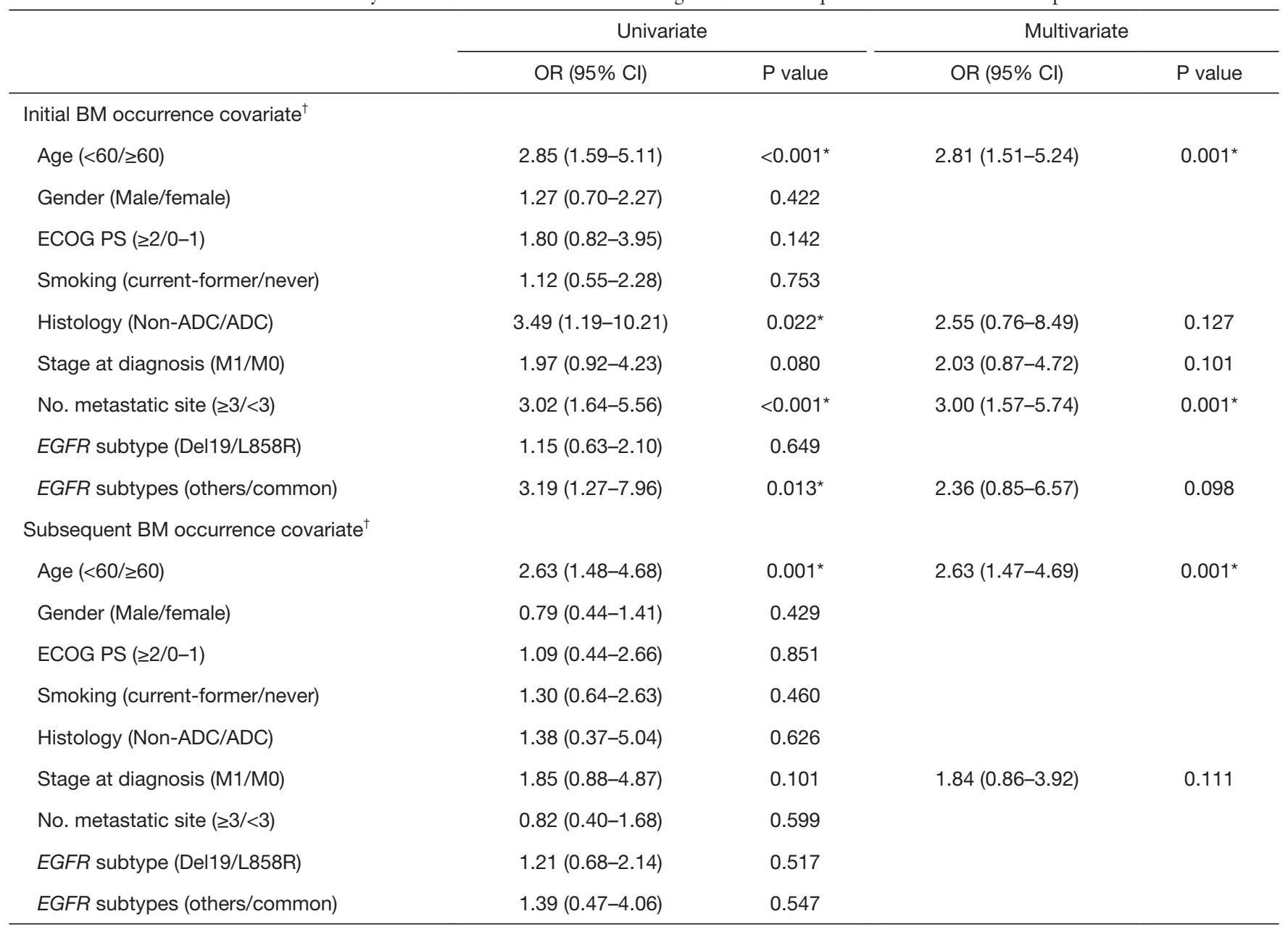

${ }^{\dagger}$, Category after the slash (/) was set as reference category. * $\mathrm{P}<0.05$. BM, brain metastasis; EGFR, epidermal growth factor receptor; ECOG PS, Eastern Cooperative Oncology Group Performance Status; ADC, adenocarcinoma; M1, metastatic disease; M0, recurrent disease; OR, odds ratio.

Overall, the mean of vimentin expression in our study was $28.5 \%$ and the distribution of vimentin expression according to $\mathrm{BM}$ status and EGFR mutation status was shown in Figure S2. Using a value of $\geq 10 \%$ positive tumor cells, vimentin expression was detected in 83 patients (43.7\%) and was found more common in patients with BM than those without $\mathrm{BM}(53.6 \%$ vs. $33.3 \%$, respectively; $\mathrm{P}=0.005)$. Although vimentin expression was similar between mutated-EGFR (40.5\%) and those with wildtype EGFR (49.3\%), respectively, we found a significant association between vimentin expression and the occurrence of BM in patients with EGFR-mutant NSCLC (52.4\% vs. $27.6 \%$, respectively; $\mathrm{P}=0.006$ ), but not in those with wild type $E G F R$ (55.9\% vs. $42.9 \%$, respectively; $\mathrm{P}=0.28$;
Figure S3).

Using multivariate analysis in patients with EGFRmutant NSCLC, the occurrence of BM was significantly associated with the expression of vimentin (OR 2.53, 95\% CI, 1.11-5.77; $\mathrm{P}=0.027$; Table 4). Moreover, vimentin expression also was statistically significantly associated with subBM occurrence (OR 3.06, 95\% CI, 1.15-8.11, $\mathrm{P}=0.025$ ) and there was a trend of association with inBM occurrence (OR 2.69, 95\% CI, 0.88-8.17, $\mathrm{P}=0.08$; Table 4). Conversely, there was no association were identified between the vimentin expression and the BM occurrence in those with wild-type EGFR (Table S4).

Furthermore, additional analysis using other methods for cut-off point of vimentin expression by receiver operating 

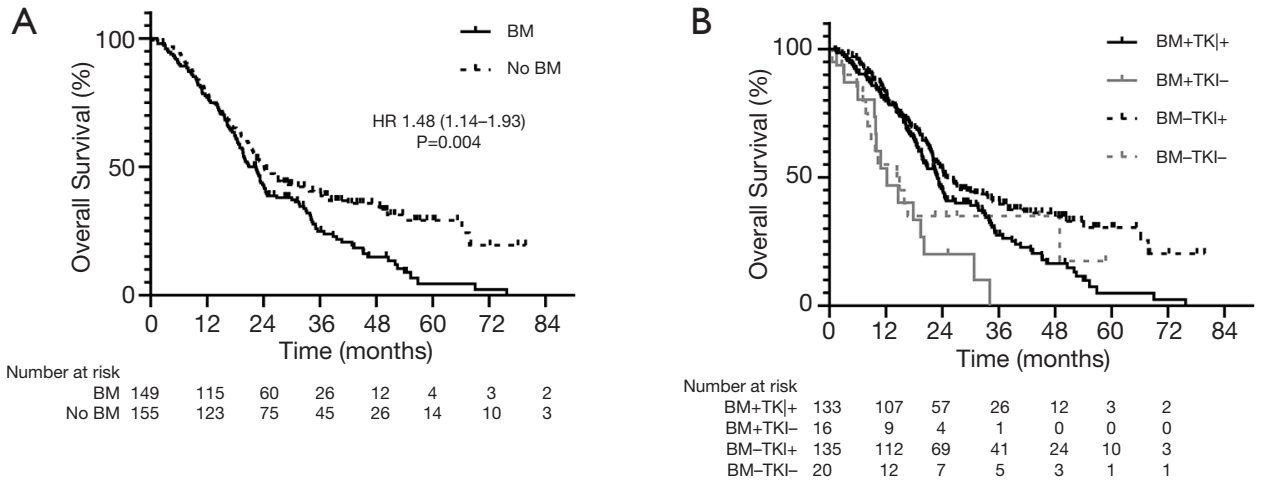

Figure 2 Outcome in patients with epidermal growth factor receptor (EGFR)-mutant NSCLC. (A) overall survival (OS) by Kaplan-Meier analysis according to brain metastasis (BM) status (B) OS by Kaplan-Meier analysis according to BM status and treatment with EGFR TKIs [patients with BM who received EGFR TKIs (BM+TKI+), patients with BM but did not receive EGFR TKIs (BM+TKI-), patients without $\mathrm{BM}$ who received EGFR TKIs (BM-TKI+), and patients without BM but did not receive EGFR TKIs (BM-TKI-)].

characteristic (ROC) analysis was also done. The area under the curve (AUC) was 0.604 (95\% CI, 0.523-0.685) and the cut-off values were $22.5 \%$. Consistent with our results that using cut-off point at $10 \%$ of positive cells, vimentin expression in EGFR-mutant but not WT significantly correlated with occurrence of BM (Figure S4, Table S5).

Taken together, these findings indicate that vimentin expression plays a role in the initiation of metastasis and promotes BM occurrence especially in patients with EGFRmutant NSCLC, and maybe serves as a potential biomarker for predicting $\mathrm{BM}$ occurrence in these patients.

\section{Prognostic role of vimentin expression in patients with NSCLC}

To determine the prognostic role of vimentin expression, we next analyzed the correlation of vimentin expression and OS according to BM and EGFR mutation status.

In the overall population regardless of BM status, tumors with positive vimentin expression tended to have shorter OS compared to those with negative expression. Median OS was 19.7 months (95\% CI, 14.23-25.19) in vimentin ${ }^{\text {positive }}$ and 22.7 months (95\% CI, 20.43-24.98) in vimentin ${ }^{\text {negative }}$, respectively $(\mathrm{P}=0.193)$. However, tumors with vimentin ${ }^{\text {positive }}$ correlated with survival according to EGFR mutation status. In the $E G F R$-mutant group, tumors with vimentin ${ }^{\text {positive }}$ had a significantly shorter OS than those with negative expression (median OS was 20.0 months (95\% CI, 14.5125.51) vs. 30.9 months (95\% CI, 20.99-40.84), respectively; HR 1.57; $\mathrm{P}=0.04)$. (Figure $3 A$ ) While there was a similar OS between positive- and negative-vimentin expression in the wild type-EGFR group. [median OS was 14.29 months (95\% CI, 10.16-18.43 months) vs. 11.86 months (95\% CI, 7.17-16.55 months), respectively; HR 0.70; $\mathrm{P}=0.18$; Figure 3B]. Moreover, in EGFR-mutant group, patients with $\mathrm{BM}$ and vimentin ${ }^{\text {positive }}$ was the worse OS compared to patients with $\mathrm{BM}$ and vimentin ${ }^{\text {negative }}$, and patients without $\mathrm{BM}$ and positive/negative vimentin expression (median OS were 16.27, 23.76, 50.40 and 38.05 months, respectively; $\mathrm{P}<0.001)$.

\section{Discussion}

Our study found a high incidence of $\mathrm{BM}$ in patients with EGFR-mutant NSCLC (49\%). Similar results were reported by previous studies (40-64\%) $(8,18,22-24)$. Furthermore, EGFR mutation not only was associated with overall BM but also predicted subBM $(9,18,19,22)$. As previously mentioned, studies on risk factors for the development of BM in NSCLC have been reported (5,6-8,10-14,2527), however, most of these studies included unselected patients with NSCLC and studies in EGFR-mutant patients were not well evaluated. Our study focused on the factors for the BM occurrence in patients with EGFR-mutant NSCLC and found the difference of risk factors between patients who experienced $\mathrm{BM}$ at diagnosis and patients who developed BM subsequently. In EGFR-mutant patients who had inBM were more likely to be younger ( $<60$ years), had non-adenocarcinoma histology, high disease burden $(\geq 3$ metastatic sites), and uncommon EGFR mutation subtype whereas only age $<60$ years was associated with subBM compared to patients without BM. Moreover, systemic 
Table 4 Vimentin expression is associated with the occurrence of BM in patients with EGFR-mutant NSCLC (N=121)

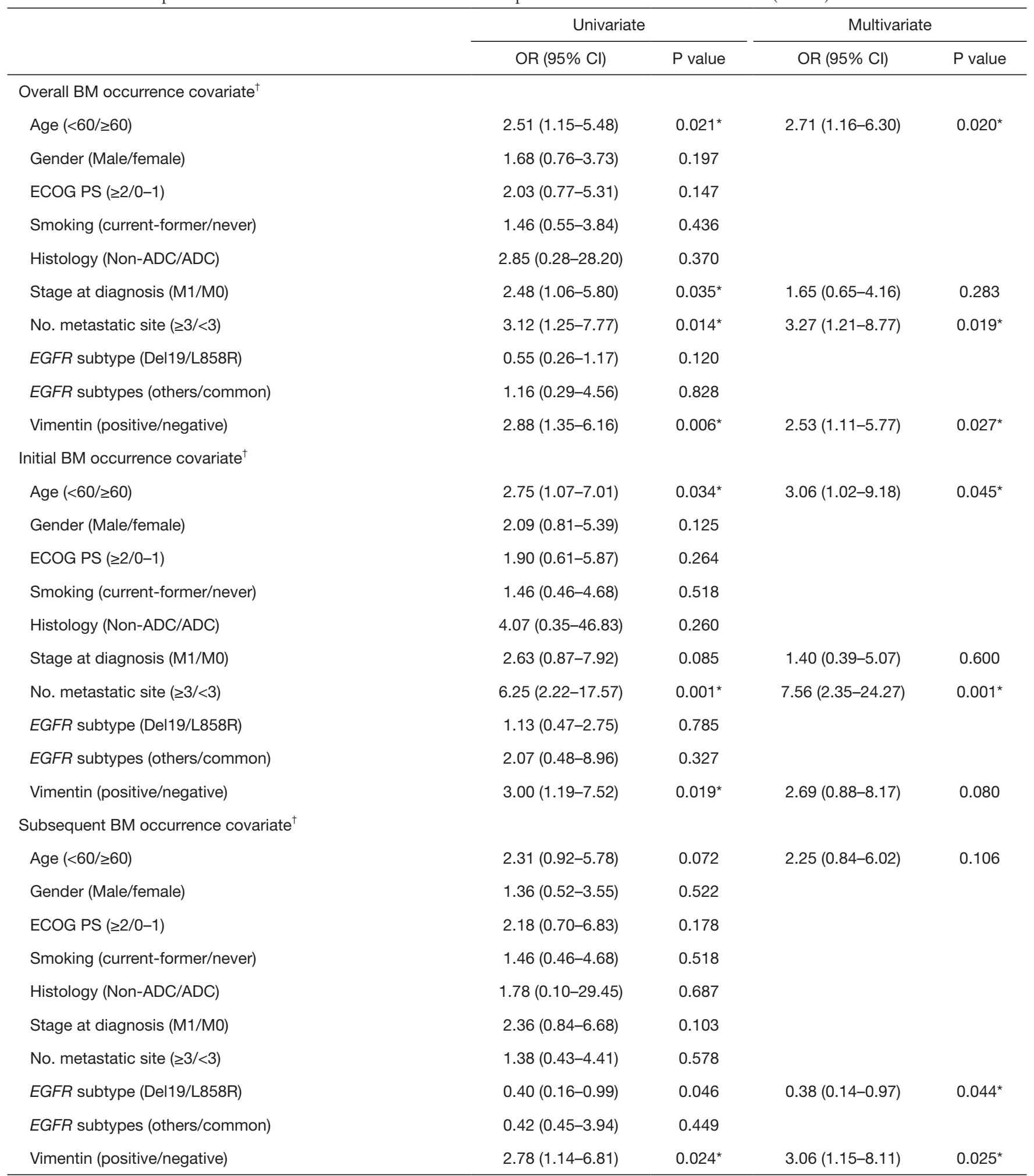

${ }^{\dagger}$, Category after the slash (/) was set as reference category. * $\mathrm{P}<0.05$. BM, brain metastasis; EGFR, epidermal growth factor receptor; NSCLC, non-small cell lung cancer; ECOG PS, Eastern Cooperative Oncology Group Performance Status; ADC, adenocarcinoma; M1, metastatic disease; $\mathrm{MO}$, recurrent disease; $\mathrm{OR}$, odds ratio. 
A

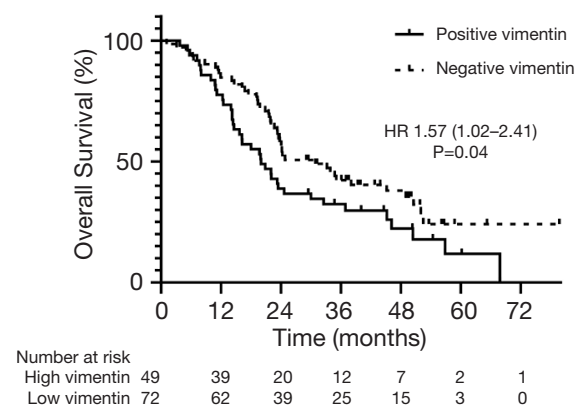

B

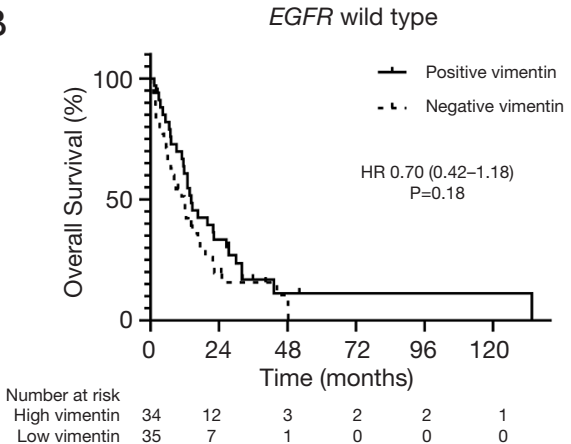

Figure 3 Prognostic role of vimentin expression in patients with NSCLC. OS by Kaplan-Meier analysis according to vimentin expression in (A) EGFR-mutant patients and (B) EGFR wild type patients

treatment may contribute to subBM in these patients. The high frequency of subBM in EGFR-mutated NSCLC can be mainly attributed to better response to systemic treatment which leads to longer survival and thus, probably increases the risk of subBM development. Our study also found an association between subBM occurrence and patients who received multiple lines of systemic treatment. We found that EGFR-TKIs could delay the occurrence of subBM more than patients who did not receive EGFR-TKIs.

It remains unclear whether the EGFR-mutation-driven cancers have biologic features that predispose towards progression and growth within the CNS. Accumulating evidence has indicated that vimentin is critical for the progression and prognosis of lung cancer (16) and preclinical studies suggested that the activation of EGFR expression promoted EMT phenotype in various cancer cell lines, including lung cancer $(17,18)$. Activating EGFR mutation enhances cell mobility and promotes vimentin expression, a hallmark of mesenchymal cells. The analyses of tumor samples revealed the association between EGFR mutation status and vimentin expression (18). Recently, AXL, a receptor tyrosine kinase belonging to the TAM (TYRO3/AXL/MER) family, and its ligand GAS6, growth arrest-specific gene 6 , has been reported to have a potential key role in various processes, including epithelial to mesenchymal transition $(28,29)$. High expression of AXL/ GAS6 has been found to be poor prognostic biomarker for NSCLC patients with BM. However, the role of EMT marker in BM from patients with EGFR-mutant NSCLC and its potential prognostic importance have not been well identified. Herein, the analyses of tumor samples from our cohort supported the correlation of vimentin expression and BM occurrence in patients with EGFR-mutated NSCLC.
Overexpression of vimentin has been observed in nearly $45 \%$ of patients in this study and found more common in patients with BM than those without BM. We also found the association between vimentin expression and the occurrence of BM especially in patients with EGFR-mutant NSCLC and conferred worse survival outcome in these patients. To best of our knowledge, this is the first study to identify the vimentin expression as one potential biomarker for poor outcome of BM among patients with EGFR-mutant NSCLC. Because this biomarker can be readily established in clinical practice using widely available IHC methods with reasonable cost, our findings may have clinical implications as this potential biomarker can predict BM occurrence and serve as a therapeutic target in these patients.

There are several limitations to our study. First, patient selection and information bias may have occurred due to the retrospective nature and single center setting of the study. Second, there was a different strategy to define patients with $\mathrm{BM}$ due to a lack of routine brain imaging in asymptomatic patients. Third, the possibility of vimentin expression discordance between the primary and metastatic sites may influence our results. There was a various cut-off point of IHC interpretation for vimentin expression when comparing our result to other studies. Therefore, interpretation should be done more carefully for future study. Finally, there are several markers related to EMT process, including epithelial markers (E-cadherin, $\mathrm{N}$-cadherin), transcription factors that repress E-cadherin expression (Snail, Twist) and mesenchymal markers (vimentin) (30). Combination assessment of these EMT-related biomarkers to explore the clinical significance of distinct EMT phenotype in these patients would add additional information.

In conclusion, younger patients with $E G F R$-mutant 
NSCLC who had high disease burden were more likely to development of BM. Vimentin serves as a biomarker predicting $\mathrm{BM}$ and poor prognostic factor in EGFR-mutant patients. Our findings may have important implications for treatment and follow-up strategies in these highrisk patients. Vimentin may be a prognostic factor and therapeutic target for $\mathrm{BM}$ in patients with EGFR mutant NSCLC.

\section{Acknowledgments}

Funding: This work was supported by The Ratchadapiseksompotch Endowment Fund [RA62/101], Faculty of Medicine, Chulalongkorn University to PS; and Chulalongkorn Academic Advancement into Its 2nd Century (CUAASC) Project to VS and CV.

\section{Footnote}

Reporting Checklist: The authors have completed the REMARK reporting checklist. Available at http://dx.doi. org/10.21037/tlcr-20-1020

Data Sharing Statement: Available at http://dx.doi. org/10.21037/tlcr-20-1020

Conflicts of Interest: All authors have completed the ICMJE uniform disclosure form (available at http://dx.doi. org/10.21037/tlcr-20-1020). The authors have no conflicts of interest to declare.

Ethical Statement: The authors are accountable for all aspects of the work in ensuring that questions related to the accuracy or integrity of any part of the work are appropriately investigated and resolved. This study was approved by the Institutional Review Board of the Faculty of Medicine at Chulalongkorn University. (No. 267/62). For this retrospective study, the written informed consent from patients was waived per the IRB, and the study was performed following the Health Insurance Portability and Accountability Act and the Declaration of Helsinki (as revised in 2013).

Open Access Statement: This is an Open Access article distributed in accordance with the Creative Commons Attribution-NonCommercial-NoDerivs 4.0 International License (CC BY-NC-ND 4.0), which permits the non- commercial replication and distribution of the article with the strict proviso that no changes or edits are made and the original work is properly cited (including links to both the formal publication through the relevant DOI and the license). See: https://creativecommons.org/licenses/by-nc-nd/4.0/.

\section{References}

1. Torre LA, Siegel RL, Jemal A. Lung Cancer Statistics. Adv Exp Med Biol 2016;893:1-19.

2. Bray F, Ferlay J, Soerjomataram I, et al. Global cancer statistics 2018: GLOBOCAN estimates of incidence and mortality worldwide for 36 cancers in 185 countries. CA Cancer J Clin 2018;68:394-424.

3. Siegel RL, Miller KD, Jemal A. Cancer statistics, 2019. CA Cancer J Clin 2019;69:7-34.

4. Schuette $W$. Treatment of brain metastases from lung cancer: chemotherapy. Lung Cancer 2004;45 Suppl 2:S253-7.

5. Shin DY, Na, II, Kim CH, et al. EGFR mutation and brain metastasis in pulmonary adenocarcinomas. J Thorac Oncol 2014;9:195-9.

6. Doebele RC, Lu X, Sumey C, et al. Oncogene status predicts patterns of metastatic spread in treatment-naive nonsmall cell lung cancer. Cancer 2012;118:4502-11.

7. Li B, Sun SZ, Yang M, et al. The correlation between EGFR mutation status and the risk of brain metastasis in patients with lung adenocarcinoma. J Neurooncol 2015;124:79-85.

8. Iuchi T, Shingyoji M, Itakura $M$, et al. Frequency of brain metastases in non-small-cell lung cancer, and their association with epidermal growth factor receptor mutations. Int J Clin Oncol 2015;20:674-9.

9. Li L, Luo S, Lin H, et al. Correlation between EGFR mutation status and the incidence of brain metastases in patients with non-small cell lung cancer. J Thorac Dis 2017;9:2510-20.

10. Ceresoli GL, Reni M, Chiesa G, et al. Brain metastases in locally advanced nonsmall cell lung carcinoma after multimodality treatment: risk factors analysis. Cancer 2002;95:605-12.

11. Bajard A, Westeel V, Dubiez A, et al. Multivariate analysis of factors predictive of brain metastases in localised nonsmall cell lung carcinoma. Lung Cancer 2004;45:317-23.

12. Hsiao SH, Chung CL, Chou YT, et al. Identification of subgroup patients with stage IIIB/IV non-small cell lung cancer at higher risk for brain metastases. Lung Cancer 2013;82:319-23. 
13. Ji Z, Bi N, Wang J, et al. Risk factors for brain metastases in locally advanced non-small cell lung cancer with definitive chest radiation. Int J Radiat Oncol Biol Phys 2014;89:330-7.

14. Mujoomdar A, Austin JH, Malhotra R, et al. Clinical predictors of metastatic disease to the brain from nonsmall cell lung carcinoma: primary tumor size, cell type, and lymph node metastases. Radiology 2007;242:882-8.

15. Eichler AF, Chung E, Kodack DP, et al. The biology of brain metastases-translation to new therapies. Nat Rev Clin Oncol 2011;8:344-56.

16. Ye Z, Zhang X, Luo Y, et al. Prognostic Values of Vimentin Expression and Its Clinicopathological Significance in Non-Small Cell Lung Cancer: A MetaAnalysis of Observational Studies with 4118 Cases. PLoS One 2016;11:e0163162.

17. Lo HW, Hsu SC, Xia W, et al. Epidermal growth factor receptor cooperates with signal transducer and activator of transcription 3 to induce epithelial-mesenchymal transition in cancer cells via up-regulation of TWIST gene expression. Cancer Res 2007;67:9066-76.

18. Hsiao SH, Chou YT, Lin SE, et al. Brain metastases in patients with non-small cell lung cancer: the role of mutated-EGFRs with an exon 19 deletion or L858R point mutation in cancer cell dissemination. Oncotarget 2017;8:53405-18.

19. Ma X, Zhu H, Guo H, et al. Risk factors of brain metastasis during the course of EGFR-TKIs therapy for patients with EGFR-mutated advanced lung adenocarcinoma. Oncotarget 2016;7:81906-17.

20. Hubbs JL, Boyd JA, Hollis D, et al. Factors associated with the development of brain metastases: analysis of 975 patients with early stage nonsmall cell lung cancer. Cancer 2010;116:5038-46.

21. Richardson F, Young GD, Sennello R, et al. The evaluation of E-Cadherin and vimentin as biomarkers of clinical

Cite this article as: Teocharoen $\mathrm{R}$, Ruangritchankul K, Vinayanuwattikun C, Sriuranpong V, Sitthideatphaiboon P. Vimentin expression status is a potential biomarker for brain metastasis development in EGFR-mutant NSCLC patients. Transl Lung Cancer Res 2021;10(2):790-801. doi: 10.21037/tlcr20-1020 outcomes among patients with non-small cell lung cancer treated with erlotinib as second- or third-line therapy. Anticancer Res 2012;32:537-52.

22. Han G, Bi J, Tan W, et al. A retrospective analysis in patients with EGFR-mutant lung adenocarcinoma: is EGFR mutation associated with a higher incidence of brain metastasis? Oncotarget 2016;7:56998-7010.

23. Matsumoto S, Takahashi K, Iwakawa R, et al. Frequent EGFR mutations in brain metastases of lung adenocarcinoma. Int J Cancer 2006;119:1491-4.

24. Welsh JW, Komaki R, Amini A, et al. Phase II trial of erlotinib plus concurrent whole-brain radiation therapy for patients with brain metastases from non-small-cell lung cancer. J Clin Oncol 2013;31:895-902.

25. Hendriks LE, Smit EF, Vosse BA, Mellema WW, Heideman DA, Bootsma GP, et al. EGFR mutated non-small cell lung cancer patients: more prone to development of bone and brain metastases? Lung Cancer 2014;84:86-91.

26. Rangachari D, Yamaguchi N, VanderLaan PA, et al. Brain metastases in patients with EGFR-mutated or ALKrearranged non-small-cell lung cancers. Lung Cancer 2015;88:108-11.

27. Baek MY, Ahn HK, Park KR, et al. Epidermal growth factor receptor mutation and pattern of brain metastasis in patients with non-small cell lung cancer. Korean J Intern Med 2018;33:168-75.

28. Linger RM, Keating AK, Earp HS, et al. TAM receptor tyrosine kinases: biologic functions, signaling, and potential therapeutic targeting in human cancer. Adv Cancer Res 2008;100:35-83.

29. Shieh YS, Lai CY, Kao YR, et al. Expression of axl in lung adenocarcinoma and correlation with tumor progression. Neoplasia 2005;7:1058-64.

30. Zeisberg M, Neilson EG. Biomarkers for epithelialmesenchymal transitions. J Clin Invest 2009;119:1429-37. 


\section{Supplementary}

Table S1 The association of systemic treatment and subsequent BM occurrence among 231 patients with EGFR-mutated NSCLC without BM at diagnosis

\begin{tabular}{|c|c|c|c|c|}
\hline Covariate $^{\dagger}$ & \multicolumn{2}{|c|}{ Univariate } & \multicolumn{2}{|c|}{ Multivariate } \\
\hline Age $(<60 / \geq 60)$ & $2.63(1.48-4.68)$ & $0.001^{*}$ & $3.22(1.68-6.15)$ & $<0.001^{*}$ \\
\hline Gender (male/female) & $0.79(0.44-1.41)$ & 0.429 & & \\
\hline ECOG PS ( $\geq 2 / 0-1)$ & $1.09(0.44-2.66)$ & 0.851 & & \\
\hline Histology (non-ADC/ADC) & $1.38(0.37-5.04)$ & 0.626 & & \\
\hline Stage at diagnosis (M1/M0) & $1.85(0.88-4.87)$ & 0.101 & $1.58(0.71-3.53)$ & 0.257 \\
\hline No. metastatic site $(\geq 3 /<3)$ & $0.82(0.40-1.68)$ & 0.599 & & \\
\hline EGFR subtype (Del19/L858R) & $1.21(0.68-2.14)$ & 0.517 & & \\
\hline No. lines of treatment $(\geq 3 / 1-2)$ & $2.84(1.58-5.12)$ & $<0.001^{*}$ & $1.82(0.89-3.73)$ & 0.101 \\
\hline TKIs (no/yes) & $1.55(0.73-3.27)$ & 0.251 & & \\
\hline TKIs as first treatment (no/yes) & $2.30(1.25-4.23)$ & $0.007^{\star}$ & $1.77(0.86-3.67)$ & 0.119 \\
\hline Generation of TKIs(first/others) & $2.32(0.65-8.34)$ & 0.197 & & \\
\hline First generation of TKIs (gefitinib/erlotinib) & $1.02(0.52-2.01)$ & 0.955 & & \\
\hline Subsequent $3^{\text {rd }}$ generation TKIs (no/yes)^ & $1.14(0.18-7.40)$ & 0.889 & & \\
\hline
\end{tabular}

${ }^{\dagger}$, Category after the slash (/) was set as reference category. ${ }^{\ddagger}$, Only 101 patients who progressed after EGFR-TKIs treatment were further tested for secondary T790M mutation. * $\mathrm{P}<0.05 ; \wedge, 58$ patients received 3rd generation of EGFR TKIs as subsequent treatment. BM, brain metastasis; EGFR, epidermal growth factor receptor; NSCLC, non-small cell lung cancer; ECOG PS, Eastern Cooperative Oncology Group Performance Status; ADC, adenocarcinoma; M1, metastatic disease; M0, recurrent disease; OR, odds ratio.

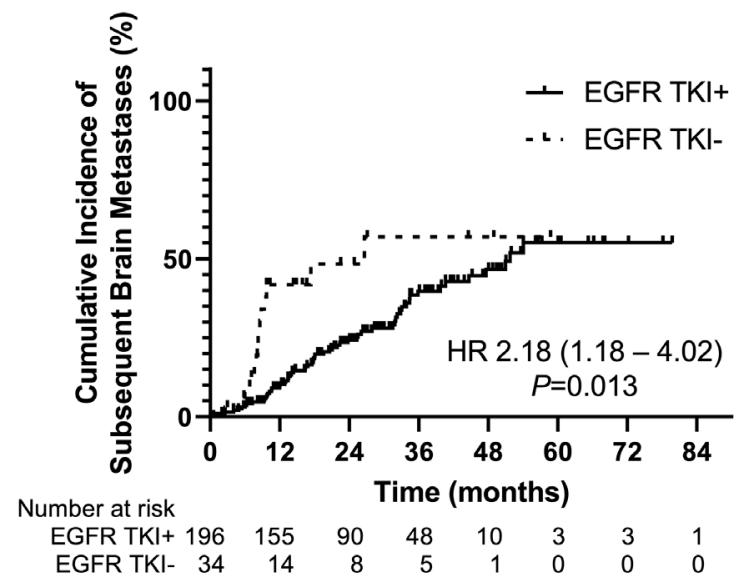

Figure S1 Estimated cumulative incidence curves illustrating subsequent brain metastasis over time according to EGFR TKIs treatment. EGFR, epidermal growth factor receptor. 
Table S2 Factors associated with time to subsequent BM (TTSBM) among 231 patients with EGFR-mutated NSCLC without BM at diagnosis

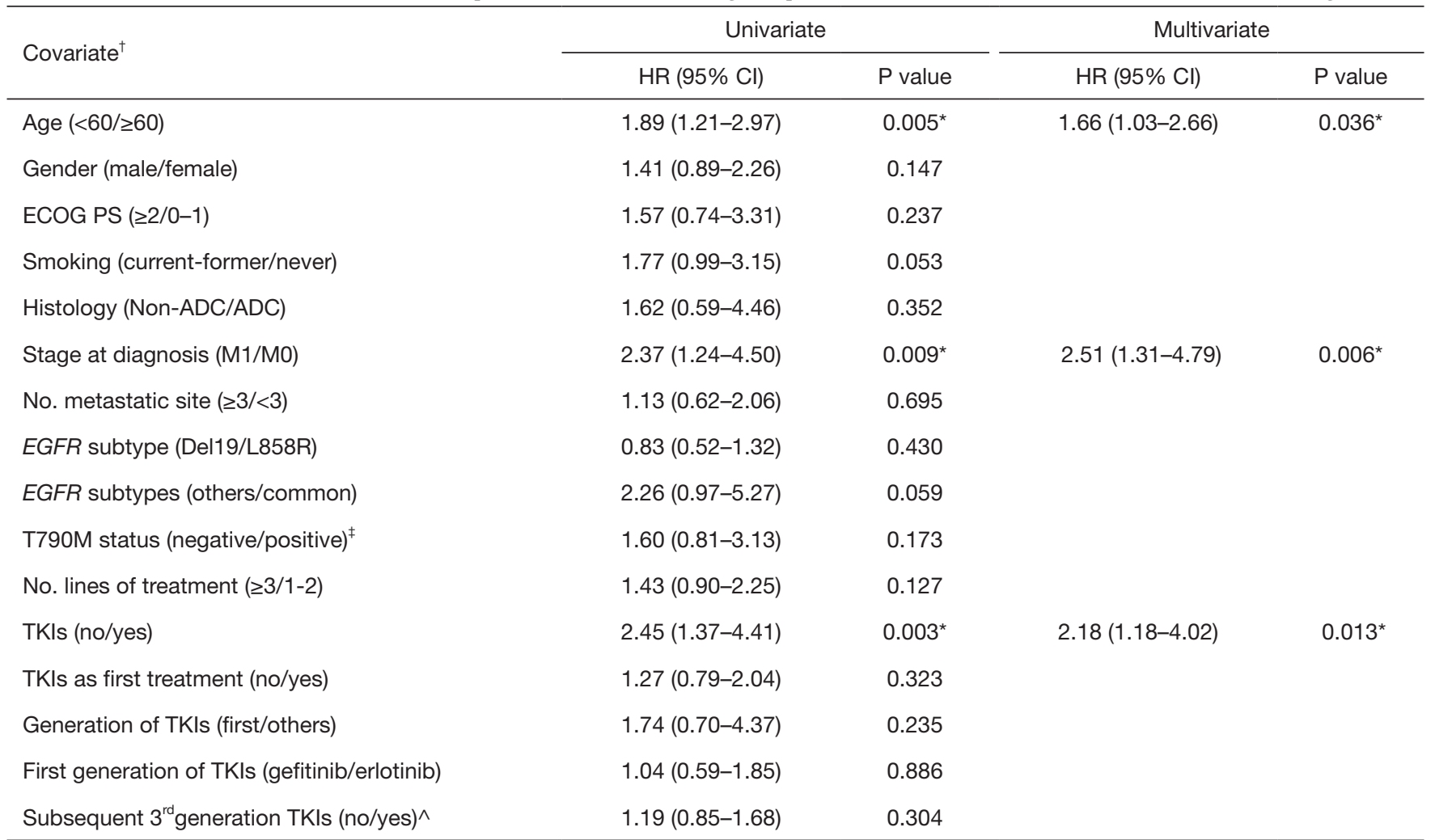

${ }^{\dagger}$, Category after the slash (/) was set as reference category. ${ }^{\ddagger}$, Only 101 patients who progressed after EGFR-TKIs treatment were further tested for secondary T790M mutation. * $\mathrm{P}<0.05$; ^, 58 patients received 3rd generation of EGFR TKIs as subsequent treatment. BM, brain metastasis; EGFR, epidermal growth factor receptor; NSCLC, non-small cell lung cancer; ECOG PS, Eastern Cooperative Oncology Group Performance Status; ADC, adenocarcinoma; M1, metastatic disease; M0, recurrent disease; HR, hazard ratio. 
Table S3 Baseline characteristics for the 190 available tumor specimens for vimentin expression by IHC according to EGFR mutation status

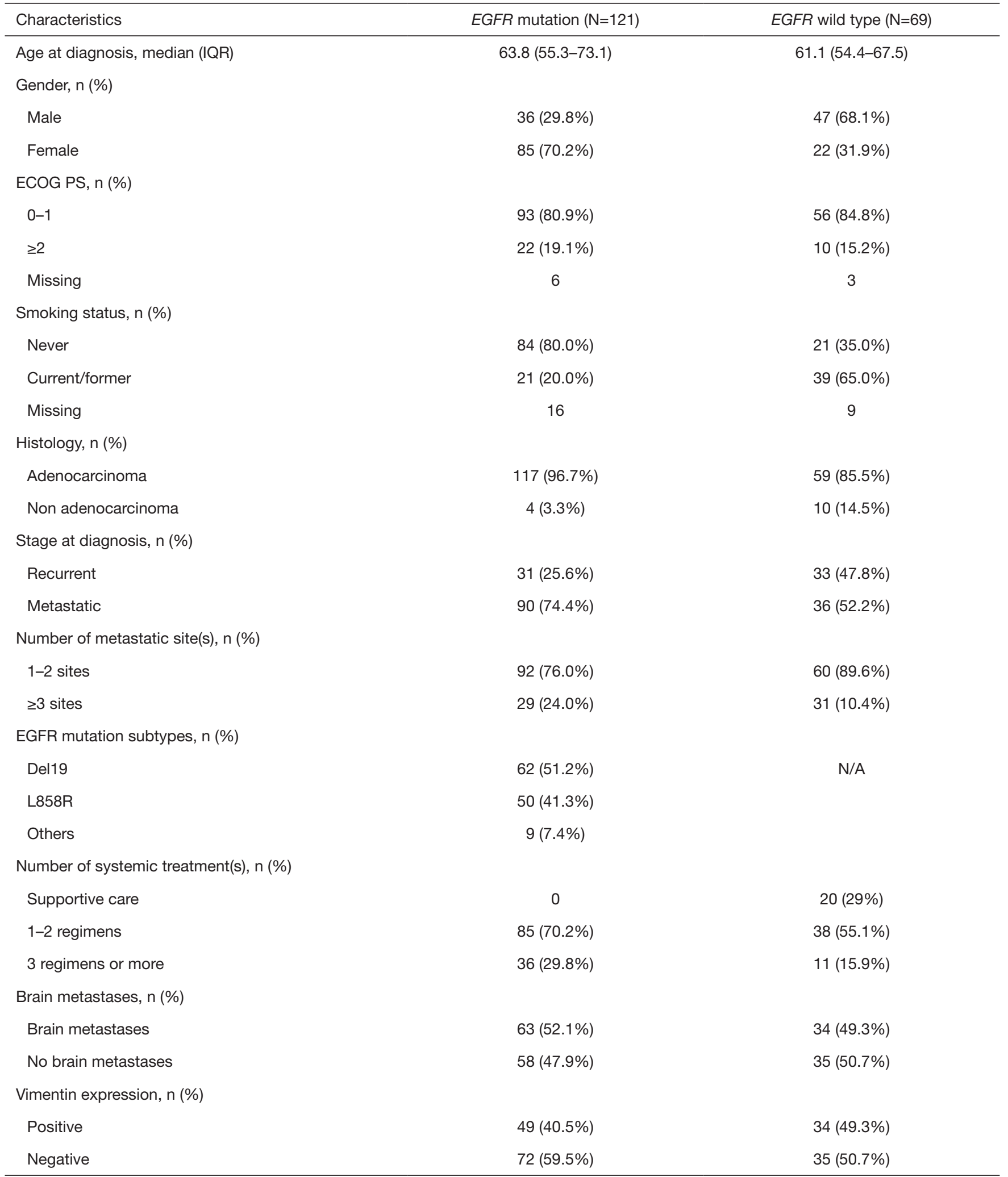

EGFR, epidermal growth factor receptor; IQR, interquartile range; ECOG PS, Eastern Cooperative Oncology Group Performance Status. 

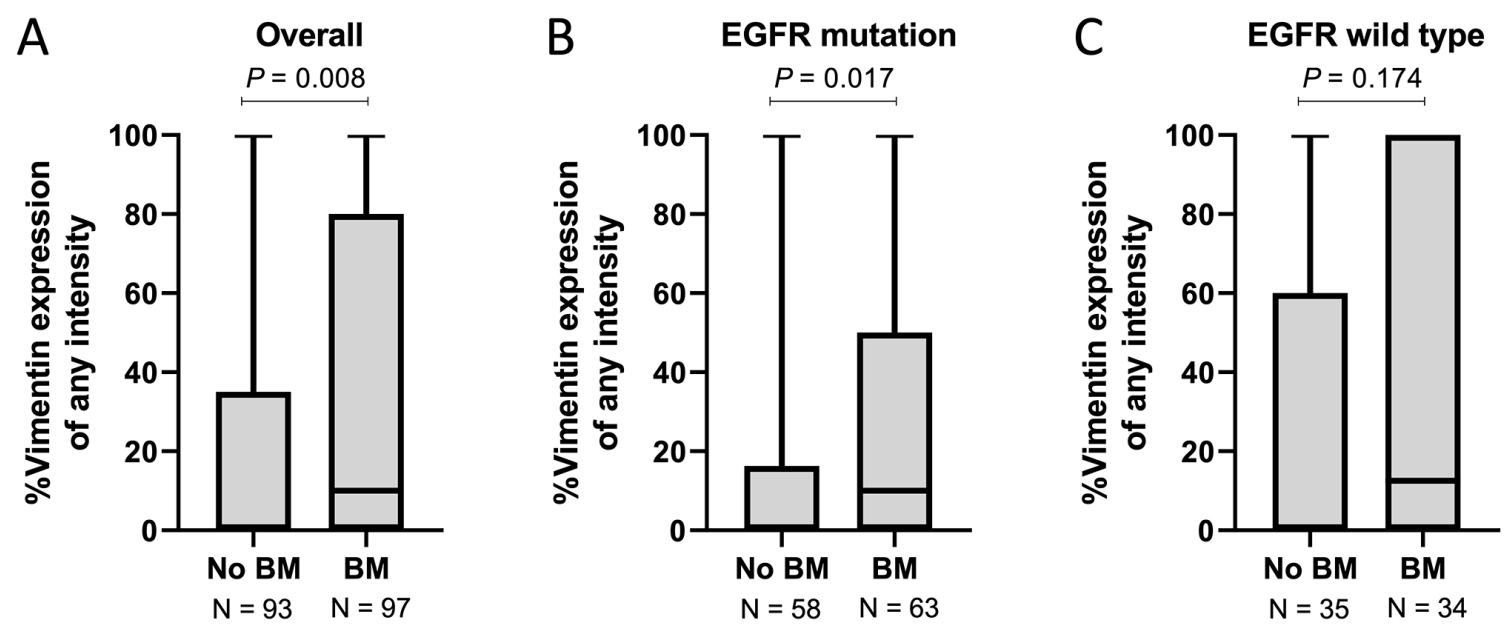

Figure S2 Distribution of vimentin expression according to BM status and EGFR mutation status in patients with NSCLC. BM, brain metastasis; EGFR, epidermal growth factor receptor; NSCLC, non-small cell lung cancer.

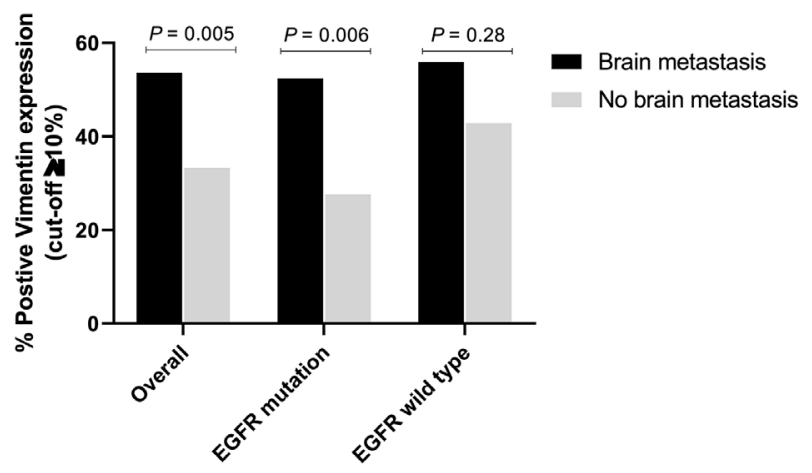

Figure S3 Correlation between vimentin expression according to BM status and EGFR mutation status in patients with NSCLC. BM, brain metastasis; EGFR, epidermal growth factor receptor; NSCLC, non-small cell lung cancer. 
Table S4 Vimentin expression is associated with occurrence of BM in patients with wild-type $E G F R(\mathrm{~N}=69)$

\begin{tabular}{lcc}
\hline & \multicolumn{2}{c}{ Univariate } \\
\hline Overall BM occurrence covariate ${ }^{\dagger}$ & OR $(95 \%$ Cl) & P value \\
\hline Age $(<60 / \geq 60)$ & $0.66(0.26-1.71)$ & 0.394 \\
Gender (Male/female) & $1.64(0.59-4.58)$ & 0.343 \\
ECOG PS ( $\geq 2 / 0-1)$ & $2.69(0.63-11.49)$ & 0.181 \\
Smoking (current-former/never) & $2.88(0.95-8.72)$ & 0.062 \\
Histology (Non-ADC/ADC) & $1.66(0.42-6.50)$ & 0.466 \\
Stage at diagnosis (M1/M0) & $0.84(0.33-2.17)$ & 0.722 \\
No. metastatic site $(\geq 3 /<3)$ & $0.40(0.07-2.23)$ & 0.295 \\
Vimentin (positive/negative) & $1.68(0.65-4.37)$ & 0.281 \\
\hline
\end{tabular}

${ }^{\dagger}$, Category after the slash (/) was set as reference category. BM, brain metastasis; EGFR, epidermal growth factor receptor; ECOG PS, Eastern Cooperative Oncology Group Performance Status; ADC, adenocarcinoma; M1, metastatic disease; M0, recurrent disease; OR, odds ratio.

\section{ROC curve}

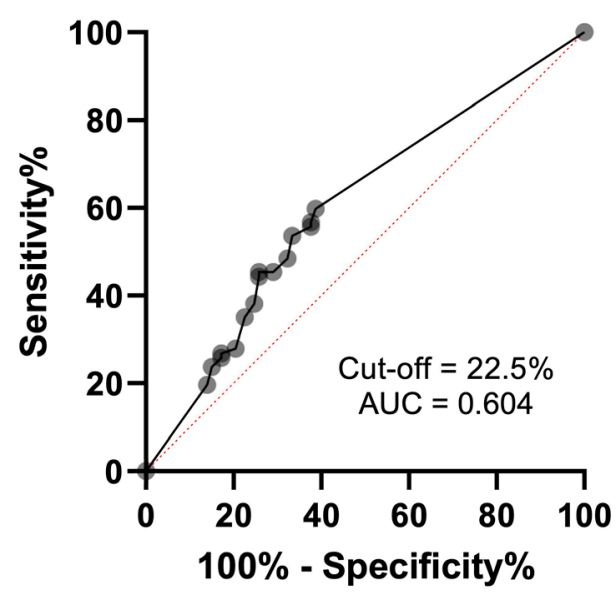

Figure S4 The cut-off value of vimentin expression using ROC curve analysis. ROC, receiver operating characteristic. 
Table S5 Vimentin expression and occurrence of BM using the cut-off value of vimentin expression by ROC analysis

\begin{tabular}{|c|c|c|c|c|}
\hline & \multicolumn{2}{|c|}{ Univariate } & \multicolumn{2}{|c|}{ Multivariate } \\
\hline \multicolumn{5}{|l|}{ Overall populations covariate $^{\dagger}$} \\
\hline Age $(<60 / \geq 60)$ & $1.46(0.81-2.62)$ & 0.210 & & \\
\hline Gender (Male/female) & $1.49(0.84-2.65)$ & 0.177 & & \\
\hline Smoking (current-former/never) & $1.73(0.91-3.28)$ & 0.095 & $1.82(0.92-3.60)$ & 0.085 \\
\hline Histology (Non-ADC/ADC) & $1.80(0.58-5.59)$ & 0.309 & & \\
\hline Stage at diagnosis (M1/M0) & $1.56(0.85-2.85)$ & 0.152 & & \\
\hline No. metastatic site $(\geq 3 /<3)$ & $0.97(0.93-4.17)$ & 0.078 & $2.42(1.03-5.69)$ & $0.042^{*}$ \\
\hline \multicolumn{5}{|l|}{ Mutant EGFR covariate $^{\dagger}$} \\
\hline Age $(<60 / \geq 60)$ & $2.51(1.15-5.48)$ & $0.021^{*}$ & $2.93(1.25-6.86)$ & $0.013^{*}$ \\
\hline Gender (Male/female) & $1.68(0.76-3.73)$ & 0.197 & & \\
\hline ECOG PS ( $\geq 2 / 0-1)$ & $2.03(0.77-5.31)$ & 0.147 & & \\
\hline Smoking (current-former/never) & $1.46(0.55-3.84)$ & 0.436 & & \\
\hline Histology (Non-ADC/ADC) & $2.85(0.28-28.20)$ & 0.370 & & \\
\hline Stage at diagnosis (M1/M0) & $2.48(1.06-5.80)$ & $0.035^{*}$ & $1.71(0.69-4.26)$ & 0.250 \\
\hline No. metastatic site $(\geq 3 /<3)$ & $3.12(1.25-7.77)$ & $0.014^{*}$ & $3.27(1.21-8.80)$ & $0.019^{*}$ \\
\hline Vimentin (positive/negative) & $3.07(1.37-6.87)$ & $0.006^{*}$ & $2.92(1.22-6.98)$ & $0.016^{*}$ \\
\hline Histology (Non-ADC/ADC) & $1.66(0.42-6.50)$ & 0.466 & & \\
\hline Stage at diagnosis (M1/M0) & $0.84(0.33-2.17)$ & 0.722 & & \\
\hline No. metastatic site $(\geq 3 /<3)$ & $0.40(0.07-2.23)$ & 0.295 & & \\
\hline Vimentin (positive/negative) & $1.70(0.65-4.49)$ & 0.282 & & \\
\hline
\end{tabular}

${ }^{\dagger}$, Category after the slash (/) was set as reference category. ${ }^{*}, \mathrm{P}<0.05$. BM, brain metastasis; ROC, receiver operating characteristic; ECOG PS, Eastern Cooperative Oncology Group Performance Status; ADC, adenocarcinoma; M1, metastatic disease; M0, recurrent disease; EGFR, epidermal growth factor receptor; OR, odds ratio. 